\title{
Enhanced surveillance to monitor response to a provincial overdose emergency, Canada
}

\author{
TIANXIN CHU*1, Sara Forsting ${ }^{1}$, Jat Sandhu', ${ }^{1,}$, Geoff Ramler ${ }^{1}$, Shannon Riley ${ }^{1}$, \\ Miranda Compton ${ }^{1}$, Mark Lysyshyn ${ }^{1,2}$ and Patricia Daly ${ }^{1,2}$
}

${ }^{1}$ Vancouver Coastal Health, Vancouver, BC, Canada; ${ }^{2}$ The University of British Columbia, Vancouver, BC, Canada

\section{Objective}

To describe the use of multiple data sources to monitor overdoses in near real-time in order to evaluate response to the provincial overdose emergency

\section{Introduction}

On April 14, 2016, British Columbia (BC)'s Provincial Health Officer declared a public health emergency due to a significant increase in drug-related overdoses and deaths in the Province. Despite the declaration, 161 suspected drug overdose deaths were reported across the Province in December 2016, a 137\% increase over the number of deaths occurring in the same month of 2015 [1]. In response to the surge overdoses, Vancouver Coastal Health Authority (VCH), one of 5 health regions within $\mathrm{BC}$, rapidly implemented a number of novel harm reduction initiatives. Overdose Prevention Sites (OPS) were opened on December 8, 2016. At these sites, people using illicit drugs are supervised by peers who can provide rapid intervention if an overdose occurs. The Mobile Medical Unit (MMU), a temporary state-of-art medical facility, was deployed on December 13, 2016 to reduce the congestion for the BC Ambulance Service (BCAS) and a major urban emergency department (ED) [2]. Following deployment of the MMU, services were transitioned to a permanent program at the Downtown Eastside Connections Clinic (DTES Connections) in the spring of 2017. DTES Connections was created to provide rapid access to addiction treatment [3]. In order to keep pace with the rapidly increasing number of novel harm reduction initiatives, enhanced surveillance programs were implemented at $\mathrm{VCH}$ to monitor and evaluate these innovative harm reduction activities, including development of new surveillance programs for the MMU, OPS and DTES Connections, along with existing routine surveillance system from EDs and a Supervised Injection Site (Insite).

\section{Methods}

Since 2011, after a spike of heroin-related deaths was reported in the Vancouver region, $\mathrm{VCH}$ started weekly monitoring of overdoses at nine EDs and Insite. Daily data extracts from EDs are automatically transferred to a secure driver by secure file transfer protocols. Groups of ICD 9/10 codes and keywords were refined to identify overdoses from EDs. A formal epidemiological evaluation was conducted to measure the algorithm's accuracy in 2013. A live connection with Insite database was set up in 2011. Overdose events at Insite are clinically determined by clinical staff. Substance injected, characteristics of overdose event and emergency interventions are entered in the database.

With the implementation of MMU, OPS and DTES Connections, a series of protocols were developed to monitor visitors' information and overdose events from each site. Demographic information, visit information, clinical presentations and substance used are collected from MMU and DTES Connections. A subset of data fields, including client handle, visit information, substance involved, overdose occurrence, naloxone intervention and ED transfer, are collected from OPS to minimize impact on peers and community partners who run the sites.

\section{Results}

Between November 2016 and January 2017, a sharp increase in overdoses was identified from EDs and Insite. Opioids, especially fentanyl and analogs, most likely contributed to the sudden increase. Weeks with government income assistance payment showed an even greater increase in overdoses.

Since December 2016, six OPS opened in Vancouver. Four of them are still operating and one received federal approval to become a supervised consumption site. By September 2017, there were 184,760 visits to the OPS. 1,017 overdoses were reversed.

A total of 2,798 patients visited the MMU during the whole operation period. $589(21 \%)$ presented from treatment of overdose. The highest number of overdose visits occurred on December 21, 2016 after that month's income assistance payment. Since then, the number of visits fluctuated with most visits driven by non-overdose related reasons. $89 \%$ of overdose visits arrived by BCAS and $79 \%$ of overdoses needed emergent and urgent care.

108 patients were transferred to DTES Connections by BCAS for treatment of overdoses by September 2017. All patients presented with opioid addiction issues.

As of the end of September 2017, no deaths were reported from OPS, MMU and DTES Connections since operations.

\section{Conclusions}

As $\mathrm{VCH}$ continues responding to the drug overdose emergency in face of increasing drug overdoses, enhanced surveillance data have been widely used by the VCH Emergency Overdose Response Committee for decision making on harm reduction activities, such as expanding operation hours at OPS and Insite on income assistance payment days; examining the impact on EDs of opening the MMU; encouraging users to avoid using alone; opening new supervised injection service and women's only OPS; and referring ED patients with non-fatal overdose to rapid access opioid agonist treatment and outreach follow-up.

\section{Keywords}

Overdose; Opioid; Drug overdose surveillance; Enhanced surveillance; Syndromic Surveilance

\section{Acknowledgments}

We would like to acknowledge the support from all of the partner organizations that contribute to the new initiatives, including Provincial Health Services Authority and community partners, and the support from site managers, nurses, outreach workers and peers to assist with the data collection.

\section{References}

1. B.C. Coroners Service monthly report. Available from http://www2. gov.bc.ca/assets/gov/public-safety-and-emergency-services/deathinvestigation/statistical/illicit-drug.pdf

2. Mobile Medical Unit Opens in Downtown Eastside to Treat Overdose Victims. Available from http://www.vancourier.com/news/mobilemedical-unit-opens-in-downtown-eastside-to-treat-overdosevictims-1.4364941

3. First-of-its-kind drug treatment centre opening in Downtown Eastside. Available from http://vancouversun.com/news/local-news/first-of-itskind-drug-treatment-centre-opening-in-downtown-eastside

\section{*TIANXIN CHU}

E-mail: chu_career@hotmail.com 